INSTITUT NATIONAL DE RECHERCHE EN INFORMATIQUE ET EN AUTOMATIQUE

\title{
On the behavior of upwind schemes in the low Mach number limit : II. Godunov type schemes.
}

\author{
Hervé Guillard — Angelo Murrone
}

$\mathbf{N}^{\circ} 4189$

Mai 2001

THÈME 4 



\title{
On the behavior of upwind schemes in the low Mach number limit : II. Godunov type schemes.
}

\author{
Hervé Guillard* , Angelo Murrone ${ }^{\dagger}$ \\ Thème 4 - Simulation et optimisation \\ de systèmes complexes \\ Projet SMASH \\ Rapport de recherche $\mathrm{n}^{\circ} 4189$ - Mai 2001 - 23 pages
}

\begin{abstract}
This paper presents an analysis of Godunov scheme in the low Mach number regime. We study the Riemann problem and show that the interface pressure contains acoustic waves of order Mach even if the initial data are well prepared and contain only pressure fluctuations of order Mach squared. We then propose to modify the fluxes computed by Godunov type schemes by solving a preconditioned Riemann problem instead of the original one. This strategy is applied to VFRoe solvers where we show that it allows to recover a correct scaling of the pressure fluctuations. Numerical experiments confirm these theoretical results.
\end{abstract}

Key-words: Compressible flow solvers, Low Mach number, Riemann problem, Godunov scheme, VFRoe scheme, Preconditioning.

* Herve.Guillard@sophia.inria.fr - Projet SMASH

$\dagger$ Angelo.Murrone@sophia.inria.fr - Projet SMASH et DTP/STH/LMTA du CEA Cadarache, 13108 Saint-Paul-Lez-Durance Cedex (France) 


\section{Comportement des schémas décentrés dans la limite des faibles nombres de Mach : II. Schémas de type Godunov.}

Résumé : Cet article présente une analyse du comportement du schéma de Godunov dans la limite des faibles nombres de Mach. Nous étudions le problème de Riemann et montrons que la pression à l'interface entre deux cellules contient des ondes acoustiques d'ordre Mach même si les données initiales sont bien préparées et ne contiennent que des fluctuations de pression d'ordre Mach au carré. Nous proposons alors de modifier les flux calculés par les schémas de type Godunov en résolvant un problème de Riemann préconditionné au lieu du problème de Riemann original. Cette stratégie est appliquée à un solveur de type VFRoe et nous montrons qu'elle permet de retrouver un bon comportement de la pression. Des expériences numériques viennent confirmer ces résultats théoriques.

Mots-clés : Solveurs pour les écoulements compressibles, Faible nombre de Mach, Problème de Riemann, Schéma de Godunov, Schéma VFRoe, Préconditionnement. 


\begin{abstract}
Aknowledgements
The work of Angelo Murrone has been supported by a grant of the "Conseil Regional" of region PACA and the DTP/STH/LMTA of CEA Cadarache. Angelo Murrone wishes to aknowledge the support of M. Grandotto (CEA Cadarache), P. Gubernatis (CEA Cadarache) and R. Saurel (IUSTI Marseille). He would also like to thank his family for their constant support as well as C. Viozat for her precious advice.
\end{abstract}




\section{Contents}

1 Introduction 6

2 The continuous problem 8

3 The Riemann problem in the low Mach number limit 11

4 The effect of preconditioning 15

5 Conclusion $\quad 19$

6 Annex I 20 


\section{List of Figures}

1 Isovalues of the pressure, on a $\mathbf{3 1 1 4}$ node mesh for $M_{\infty}=0.1$ (top), $M_{\infty}=0.01$ (middle), $M_{\infty}=0.001$ (bottom) and for Roe scheme (left), VFRoe scheme (middle), Godunov scheme (right) $\ldots \ldots \ldots \ldots \ldots \ldots \ldots \ldots$

2 The four possible wave patterns in the solution of the Riemann problem : (a) 1rarefaction, contact, 3-shock, (b) 1-shock, contact, 3-rarefaction, (c) 1-rarefaction,

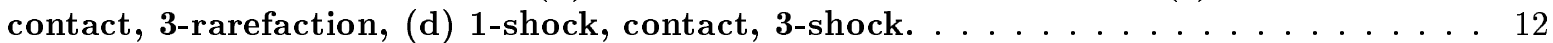

3 Intersection of rarefaction curves for $u_{R, 1}-u_{L, 1}>0$ (left) and shock curves for $u_{R, 1}-$

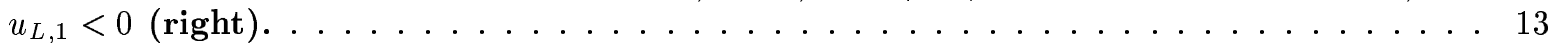

4 Comparison between Godunov solver and asymptotic analysis for $u_{R}>u_{L}$ (left) and

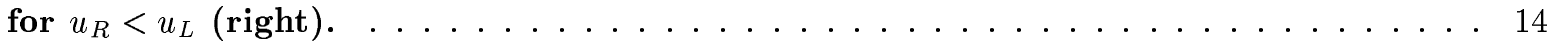

5 Pressure fluctuations vs inflow Mach number ; for comparison the curve $y=x^{*} \mathrm{x}$ is

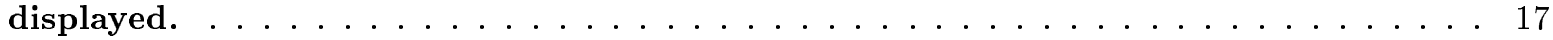

6 Isovalues of the pressure, on a 3114 node mesh for $M_{\infty}=0.1$ (top), $M_{\infty}=0.01$

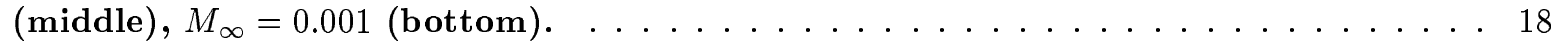




\section{Introduction}

Incompressible flows are a particular case of compressible ones and therefore in principle, a compressible flow solver should be able to compute these flows. Unfortunately, there are experimental evidences showing that on a fixed mesh, the solutions of the compressible flow discretized equations are not an accurate approximation of the solutions of the incompressible model (e.g see [26]). This question has received a considerable interest in the last years and several recent works have tried to explain the reasons of this difficulty and to construct numerical schemes valid for all Mach numbers. Some of these works extend to the compressible regime, the numerical methods used for the computation of incompressible flows. Examples of these type of methods are for instance [1] or [27]. Another approaches rely on some modifications of high order shock capturing techniques. These approaches are for instance described in [7], [2] for Roe discretization, in [3] for the HLLE scheme and in [25] for Flux schemes. Their principal ingredient is the use of preconditioning techniques originally developped for steady state computations [20], [21], [4] that are here selectively applied only to the upwind artifical viscosity.

In [7], we have examined this technique for Roe type solvers where the numerical flux between two cells takes the following form :

$$
\Phi\left(q_{L}, q_{R}, \mathbf{n}_{L R}\right)=\frac{1}{2}\left(\mathbf{F} \cdot \mathbf{n}_{L R}\left(q_{L}\right)+\mathbf{F} \cdot \mathbf{n}_{L R}\left(q_{R}\right)+\left|\frac{\partial \mathbf{F} \cdot \mathbf{n}_{L R}}{\partial q}\right| \Delta_{L R} q\right)
$$

Here $\mathbf{n}_{L R}$ is the unit normal at the interface and $\Delta_{L R} q=q_{L}-q_{R}$ is the jump between the values $q_{L}$ and $q_{R}$ on each side of the interface.

By performing an asymptotic analysis of the discrete equations, we identified the limit equations satisfied by Roe discretization and showed that these limit equations support pressure fluctuations of the order of the Mach number. Therefore, in the low Mach number limit, the solutions of the Roe compressible solver cannot be an accurate approximation of the incompressible system where the pressure fluctuations scales with the square of the Mach number.

In this paper, we examine the same problem for Godunov type schemes. In contrast with Roe type solvers, the flux here is of the form :

$$
\Phi\left(q_{L}, q_{R}, \mathbf{n}_{L R}\right)=\mathbf{F} \cdot \mathbf{n}_{L R}\left(q_{L R}\right)
$$

where $q_{L R}$ is the solution of an exact or approximate Riemann problem defined by the two states $q_{L}$ and $q_{R}$.

These type of schemes exhibit the same problem of accuracy than Roe's and other upwind schemes.

Fig. 1 shows an example of this behavior. On this figure is shown a sequence of computations of the flow around a NACA0012 airfoil at decreasing Mach number on the same mesh. In these computations, the inflow velocity is kept constant equal to unity while the inflow pressure is increased. The figures show the normalized pressure $p-p_{\min } / p_{\max }-p_{\min }$. As the Mach number decreases, the results become worse and the solutions do not converge to a reasonable approximation of the incompressible solution. The figures display from left to right the results obtained with Roe scheme where the flux is of the form (1), together with the results obtained by the VFRoe scheme (to be described below) and Godunov scheme where the fluxes are of the form (2). It is seen that the same results are obtained for these three upwind schemes.

Understanding the reason of this behavior is particularly interesting in the case of Godunov scheme. Since in this scheme, the flux computed at the interface is exact, the only error source results from the projection of the solution on the basis of piecewise constant functions. As for Roe scheme, we will see that the analysis reveals that the discretized solutions are polluted by pressure fluctuations of order Mach. Therefore, the question becomes to identify the mechanism that creates pressure waves of order Mach at the interface from an initial field that contains only pressure fluctuations of order Mach squared. 

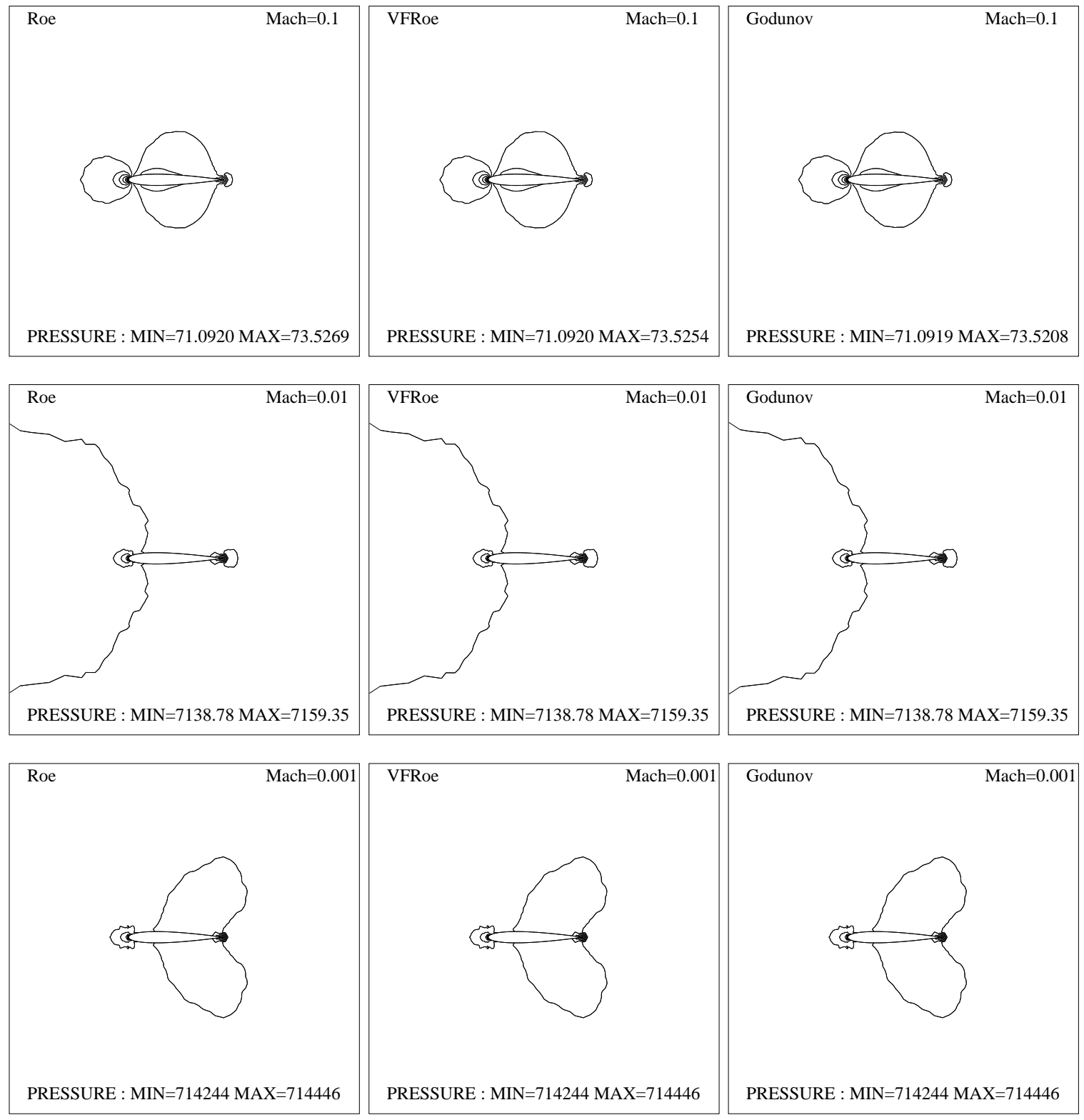

Figure 1: Isovalues of the pressure, on a 3114 node mesh for $M_{\infty}=0.1$ (top), $M_{\infty}=0.01$ (middle), $M_{\infty}=0.001$ (bottom) and for Roe scheme (left), VFRoe scheme (middle), Godunov scheme (right).

Since the work of S. Schochet [17] (see also [5]) the behavior when the Mach number goes to zero of the solutions of the compressible Euler equations for general initial data, is well understood at least for the isentropic equations : the limit solution separates into an acoustic wave that depends on a fast time variable $t / M_{*}$ plus a slow part that does not depend on this fast time variable. Moreover the slow part does satisfy the incompressible Euler equations. For a certain class of initial data (the well prepared case) the acoustic component is not present at leading order and the solutions of the incompressible Euler equations are the strong limits of the solutions of the compressible ones as was known from some earlier results of [8] and [9].

For the non-isentropic model, the situation is less clear. In the well prepared case, as shown in [16], it is known that the solutions of the incompressible model are strong limits of the solution of the compressible model. The same result is true and not necessary for well prepared data in the whole space [11]. However, in the periodic case, a lot of questions remain open (see [12] for an up-to-date review of this problem). 
The understanding of this complex behavior is useful to catch the reasons why Godunov scheme fails to compute the incompressible limit. Actually, as we will see in section 3, the interface values computed by Godunov scheme contain a fast acoustic component that comes from the projection of the initial data on piecewise constant fields. The pressure perturbations associated with this component are of order Mach and hide completely the incompressible part. A consequence of this fact is that the field computed by the discrete scheme results from a balance of acoustic waves instead of satisfying the equations of the incompressible model.

The summary of this work is as follows. In section 2 we recall some known results on the behavior of the solutions of the Euler equations in the low Mach number limit. The purpose of this section is to show that the solutions of the incompressible model are only weak limits of the solutions of the compressible Euler model. For this, we introduce a simple linear model that displays the same type of behavior than the Euler equations and we indicate how the results on this model extend to the non linear Euler model.

In section 3, we study the solutions of the Riemann problem in the low Mach number limit. We show that even if the initial data are well prepared and close to an incompressible field with pressure fluctuations of order Mach squared, the solution of the Riemann problem exhibits acoustic fluctuations that scale with the Mach number. This phenomena can be related to the fact that the projection of the initial data on piecewise constant functions creates artificial discontinuities that are responsible for the generation of acoustic waves as in the case of not well prepared data.

For the Roe scheme, it is known that the use of preconditioning is a powerful remedy to cure the accuracy problem [7], [2], the same conclusions have been reached for other types of upwind schemes as the HLLE scheme [3] or the VFFC type schemes [25]. In section 4, we study the application of preconditioning to VFRoe type schemes [10]. We show that as for the previously cited upwind schemes, preconditioning is also a powerful remedy that allows the accurate computations of near incompressible solutions.

\section{The continuous problem}

To help the understanding of the behavior of upwind schemes in the low Mach number limit, we recall in this section some known results on the continuous problem. We begin with the isentropic system where the mathematical theory is almost complete and restrict the discussion to the periodic case.

(Note that in the case of the whole space and even for general initial data, the results are stronger due to the decay at infinity of the energy of the acoustic component. As shown in [22] the limit solutions of the compressible equations satisfy the equations of the incompressible Euler model although uniform convergence breaks near $t=0$.)

The isentropic Euler equations write :

$$
\begin{array}{lll}
\frac{\partial \rho}{\partial t} & +\rho \operatorname{div} \mathbf{u} & +\mathbf{u} . \nabla \rho=0 \\
\frac{\partial \mathbf{u}}{\partial t} & +\operatorname{div}(\mathbf{u} \otimes \mathbf{u}) & +\frac{1}{\varepsilon^{2}} \frac{1}{\rho} \nabla p=0
\end{array}
$$

In these equations, $\mathbf{u}=(u, v)$ is a vector field and $\rho$ a scalar field, defined on $\mathbb{R} \times T^{2}$ where $T^{2}$ is a two dimensional periodic box. $\varepsilon$ is a small parameter proportional to the Mach number. The pressure $p$ is related to the density by the isentropic state law $p=$ Cte $\rho^{\gamma}$. To make the analysis simpler, we perform the following change of variable :

$$
\tilde{r}=\frac{2}{\gamma-1} \sqrt{p^{\prime}(\rho)}=\frac{1}{\tilde{\gamma}} \sqrt{p^{\prime}(\rho)}
$$

and set $\tilde{r}=r_{0}+\varepsilon r$ where $r_{0}$ is a constant. In term of the new variables $(r, \mathbf{u})$, the system (4) takes the form :

$$
\begin{array}{lll}
\frac{\partial r}{\partial t} & +\mathbf{u} . \nabla r & +\frac{\tilde{\gamma} r_{0}}{\varepsilon} \operatorname{div} \mathbf{u}=0 \\
\frac{\partial \mathbf{u}}{\partial t} & +\operatorname{div}(\mathbf{u} \otimes \mathbf{u})+\tilde{\gamma} r \nabla r & +\frac{\tilde{\gamma} r_{0}}{\varepsilon} \nabla r=0
\end{array}
$$


We write this system in the symbolic form :

$$
\frac{\partial q}{\partial t}+a(q, q)+\frac{\tilde{\gamma} r_{0}}{\varepsilon} L q=0
$$

with $q=(r, u, v)^{t}, L q=(\operatorname{divu}, \nabla r)^{t}$ is the linear, constant coefficient, acoustic operator.

As a first step in the analysis of (8), consider the following constant coefficient linear model problem obtained from (8) by a linearisation around the state $(r, \mathbf{u})=(0, \mathbf{a})$. For the sake of simplicity, in the following we set $\tilde{\gamma} r_{0}=1$.

$$
\begin{array}{ll}
\frac{\partial r}{\partial t} \quad+\mathbf{a} \cdot \nabla r \quad+\frac{1}{\varepsilon} \operatorname{div} \mathbf{u}=0 \\
\frac{\partial \mathbf{u}}{\partial t} \quad+\mathbf{a} \cdot \nabla \mathbf{u} \quad+\frac{1}{\varepsilon} \nabla r=0
\end{array}
$$

We write this linear system under the form :

$$
\frac{\partial q}{\partial t}+H q+\frac{1}{\varepsilon} L q=0
$$

where $H q=\mathbf{a} . \nabla q$ is a constant velocity linear advection operator. Writing (12) in Fourier space for the Fourier components $\hat{q}(\mathbf{k})$ of $q$, we get :

$$
\frac{\partial \hat{q}(\mathbf{k})}{\partial t}+i\left[\hat{H}(\mathbf{k})+\frac{1}{\varepsilon} \hat{L}(\mathbf{k})\right] \hat{q}(\mathbf{k})=0 \quad \text { for } \quad \mathbf{k} \in Z^{2}
$$

where the matrix $\hat{H}(\mathbf{k})+1 / \varepsilon \hat{L}(\mathbf{k})$ is equal to :

$$
\left(\begin{array}{ccc}
\mathbf{a . k} & k_{1} / \varepsilon & k_{2} / \varepsilon \\
k_{1} / \varepsilon & \mathbf{a . k} & 0 \\
k_{2} / \varepsilon & 0 & \mathbf{a . k}
\end{array}\right)
$$

This matrix is diagonalizable, its eigenvectors are :

$$
r_{1}(\mathbf{k})=\frac{1}{\sqrt{1+|\mathbf{k}|^{2}}}\left(\begin{array}{c}
1 \\
-k_{1} \\
-k_{2}
\end{array}\right), r_{2}(\mathbf{k})=\frac{1}{|\mathbf{k}|}\left(\begin{array}{c}
0 \\
-k_{2} \\
k_{1}
\end{array}\right), r_{3}(\mathbf{k})=\frac{1}{\sqrt{1+|\mathbf{k}|^{2}}}\left(\begin{array}{c}
1 \\
k_{1} \\
k_{2}
\end{array}\right)
$$

with associated eigenvalues $\lambda_{1}=\mathbf{a} \cdot \mathbf{k}-\frac{|\mathbf{k}|}{\varepsilon}, \lambda_{2}=\mathbf{a} \cdot \mathbf{k}$ and $\lambda_{3}=\mathbf{a} \cdot \mathbf{k}+\frac{|\mathbf{k}|}{\varepsilon}$.

Therefore, the solution of (13) is :

$$
\hat{q}(\mathbf{k}, t)=\left\{\begin{array}{l}
\frac{1}{\sqrt{1+|\mathbf{k}|^{2}}}\left(\hat{r}(\mathbf{k}, 0)-k_{1} \hat{u}(\mathbf{k}, 0)-k_{2} \hat{v}(\mathbf{k}, 0)\right) e^{-i(\mathbf{a} . \mathbf{k}-|\mathbf{k}| / \varepsilon) t} r_{1}(\mathbf{k}) \\
+\frac{1}{|\mathbf{k}|}\left(-k_{2} \hat{u}(\mathbf{k}, 0)+k_{1} \hat{v}(\mathbf{k}, 0)\right) e^{-i \mathbf{a} . \mathbf{k} t} r_{2}(\mathbf{k}) \\
+\frac{1}{\sqrt{1+|\mathbf{k}|^{2}}}\left(\hat{r}(\mathbf{k}, 0)+k_{1} \hat{u}(\mathbf{k}, 0)+k_{2} \hat{v}(\mathbf{k}, 0)\right) e^{-i(\mathbf{a} . \mathbf{k}+|\mathbf{k}| / \varepsilon) t} r_{3}(\mathbf{k})
\end{array}\right.
$$

From (16) it is seen that the solution separates into a fast oscillatory component that depends on the fast time variable $t / \varepsilon$

$$
\hat{q}_{o s c}(\mathbf{k}, t, t / \varepsilon)=\frac{1}{\sqrt{1+|\mathbf{k}|^{2}}}\left\{\begin{array}{l}
\left(\hat{r}(\mathbf{k}, 0)-k_{1} \hat{u}(\mathbf{k}, 0)-k_{2} \hat{v}(\mathbf{k}, 0)\right) e^{-i(\mathbf{a} \cdot \mathbf{k}-|\mathbf{k}| \varepsilon) t} r_{1}(\mathbf{k}) \\
+ \\
\left(\hat{r}(\mathbf{k}, 0)+k_{1} \hat{u}(\mathbf{k}, 0)+k_{2} \hat{v}(\mathbf{k}, 0)\right) e^{-i(\mathbf{a} \cdot \mathbf{k}+|\mathbf{k}| / \varepsilon) t} r_{3}(\mathbf{k})
\end{array}\right.
$$

RR $n^{\circ} 4189$ 
plus a slow component that does not depend on this fast variable :

$$
\hat{q}_{\text {slow }}(\mathbf{k}, t)=\frac{1}{|\mathbf{k}|}\left(-k_{2} \hat{u}(\mathbf{k}, 0)+k_{1} \hat{v}(\mathbf{k}, 0)\right) e^{-i \mathbf{a} \cdot \mathbf{k} t} r_{2}(\mathbf{k})
$$

In Fourier space, the kernel of the linear acoustic operator $\hat{L}(\mathbf{k})$ is the space spaned by the vector $r_{2}(\mathbf{k})=$ $\left(0,-k_{2}, k_{1}\right)^{t}$. This corresponds in physical space to the space defined by :

$$
\operatorname{ker} L=\{(r, \mathbf{u}) ; r=\text { cte, } \operatorname{div} \mathbf{u}=0\}
$$

The slow component $q_{\text {slow }}=\sum_{\mathbf{k}} \hat{q}_{\text {slow }}(\mathbf{k}, t) e^{i \mathbf{k} \cdot \mathbf{x}}$ is thus the projection of the solution on this space and represents the incompressible component of the solution. It satisfies the "incompressible system" :

$$
\frac{\partial q_{\text {slow }}}{\partial t}+H q_{\text {slow }}=0
$$

with initial data $q_{\text {slow }}(t=0)=P q(t=0)$ where $P$ is the projection on ker $L$.

From expression (17), it is seen that $q_{\text {osc }}$ converges in the sense of distributions to 0 when $\varepsilon \rightarrow 0$. However this is only a weak convergence and therefore except for a special class of initial data, the solution contains very fast oscillatory components that do not disappear when $\varepsilon \rightarrow 0$ : in general, even for very small $\varepsilon$ we have to write :

$$
q(\mathbf{x}, t)=q_{\text {slow }}(\mathbf{x}, t)+q_{o s c}(\mathbf{x}, t, t / \varepsilon)
$$

We now describe a special class of initial data such that we will have a strong convergence toward the incompressible limit. A necessary condition for $q(\mathbf{x}, t)$ to convergence strongly to the incompressible limit $q_{\text {slow }}(\mathbf{x}, t)$ is that $q_{o s c}(\mathbf{x}, t, t / \varepsilon)$ converges strongly to 0 . This is not possible except if the initial data are close to the kernel of $L$. This is the case referred to as the "well prepared case". For the isentropic Euler equations, this situation has been examined in [8] and [9] while for the non-isentropic model, we refer to [16]. if

For our model problem (11) the "well prepared case" can be described as follows. It is clear from (16) that

$$
\left\{\begin{array}{l}
\left(\hat{r}(\mathbf{k}, 0)-k_{1} \hat{u}(\mathbf{k}, 0)-k_{2} \hat{v}(\mathbf{k}, 0)\right)=\mathcal{O}(\varepsilon) \\
\left(\hat{r}(\mathbf{k}, 0)+k_{1} \hat{u}(\mathbf{k}, 0)+k_{2} \hat{v}(\mathbf{k}, 0)\right)=\mathcal{O}(\varepsilon)
\end{array}\right.
$$

then the oscillatory component of the solution $q_{o s c}$ converges strongly to 0 and thus the incompressible component $q_{\text {slow }}$ is the strong limit of $q$ as $\varepsilon \rightarrow 0$.

In physical space, the two conditions (22) mean that the initial data have the following form :

$$
\left\{\begin{array}{l}
r(\mathbf{x}, 0)=\varepsilon r_{1}(\mathbf{x}, 0) \\
\mathbf{u}(\mathbf{x}, 0)=\mathbf{u}_{0}(\mathbf{x}, 0)+\varepsilon \mathbf{u}_{1}(\mathbf{x}, 0)
\end{array}\right.
$$

where $r_{1}(\mathbf{x}, 0)$ is an $\mathcal{O}(1)$ scalar field, $\mathbf{u}_{0}(\mathbf{x}, 0)$ and $\mathbf{u}_{1}(\mathbf{x}, 0)$ two $\mathcal{O}(1)$ vector fields and $\mathbf{u}_{0}(\mathbf{x}, 0)$ satisfies divu $\mathbf{u}_{0}=0$.

Let us now return to the isentropic Euler equations (8). Although the analysis and techniques of proof are much more complex than for our linear model problem, the results are essentially the same. As proved in [17] (see also [5]), in the low Mach number limit, the solution can be written as :

$$
q(\mathbf{x}, t)=q_{\text {slow }}(\mathbf{x}, t)+q_{\text {osc }}(\mathbf{x}, t, t / \varepsilon)+\mathcal{O}(\varepsilon)
$$

and separate into a fast acoustic component $q_{o s c}(\mathbf{x}, t, t / \varepsilon)$ that depends on the fast time variable $t / \varepsilon$ and a slow part $q_{\text {slow }}(\mathbf{x}, t)$. Moreover, the slow part is solution of the incompressible equations :

$$
\begin{aligned}
& \operatorname{div}(\mathbf{u})=0 \\
& \rho_{0}\left(\frac{\partial}{\partial t} \mathbf{u} \quad+\operatorname{div}(\mathbf{u} \otimes \mathbf{u})\right)+\nabla \pi \quad=0
\end{aligned}
$$


In general $q_{\text {slow }}(\mathbf{x}, t)$ is only a weak limit of the solutions except for well prepared initial data close to the kernel of $L$. As for the model problem, this special class of initial data is characterized by :

$$
\left\{\begin{array}{l}
r(\mathbf{x}, 0)=\varepsilon r_{1}(\mathbf{x}, 0) \\
\mathbf{u}(\mathbf{x}, 0)=\mathbf{u}_{0}(\mathbf{x}, 0)+\varepsilon \mathbf{u}_{1}(\mathbf{x}, 0) \quad \text { with } \quad \operatorname{div}_{0}=0
\end{array}\right.
$$

Going back to the original variables $(p, \mathbf{u})$, we see that this implies that the initial data are such that :

$$
\left\{\begin{array}{l}
p(\mathbf{x}, 0)=\text { Cte }+\varepsilon^{2} p_{2}(\mathbf{x}, 0) \\
\mathbf{u}(\mathbf{x}, 0)=\mathbf{u}_{0}(\mathbf{x}, 0)+\varepsilon \mathbf{u}_{1}(\mathbf{x}, 0) \quad \text { with } \quad \operatorname{div} \mathbf{u}_{0}=0
\end{array}\right.
$$

where $p_{2}(\mathbf{x}, 0), \mathbf{u}_{0}(\mathbf{x}, 0), \mathbf{u}_{1}(\mathbf{x}, 0)$ are smooth, regular functions.

\section{The Riemann problem in the low Mach number limit}

We are interested in computing flows close to the incompressible limit. As seen in the previous section, if the initial data are well prepared and of the form (27) with pressure fluctuations of the order of the square of the Mach number, we have strong convergence of the solution of the compressible equations to the solution of the incompressible equations. However even if the initial data do verify this assumption, the projection of these data on piecewise constant functions creates discontinuities at the interface between the cells. When solving the Riemann problem, these discontinuities generate pressure waves of order Mach. As a consequence, instead of computing an approximation of the solution of the incompressible equations, upwind schemes compute a balance of acoustic waves.

Consider the Riemann problem defined by :

$$
\begin{aligned}
& \frac{\partial q}{\partial t}+\frac{\partial F(q)}{\partial x}=0 \\
& q(x, 0)=\left\{\begin{array}{lll}
q=q_{L} & \text { if } & x<0 \\
q=q_{R} & \text { if } & x>0
\end{array}\right.
\end{aligned}
$$

with

$$
q=\left[\begin{array}{l}
\rho \\
\rho u \\
\rho v \\
\rho e
\end{array}\right] \quad F(q)=\left[\begin{array}{l}
\rho u \\
\rho u^{2}+p \\
\rho u v \\
(\rho e+p) u
\end{array}\right]
$$

In order to make easier the description of this problem in the low Mach number limit, we recall below how to solve it in the general case. For details, one can refer for instance to ([18], [6]). The solution consists of 4 different constant states $q_{L}, q_{L}^{*}, q_{R}^{*}, q_{R}$ connected by three waves that can be from left to right, a 1-rarefaction or a 1-shock, a contact discontinuity and a 3 -rarefaction or a 3 -shock. The four possible wave patterns are represented on Fig.2.

Through a 1-rarefaction, the entropy, the tangential velocity and the quantity $u+2 a /(\gamma-1)$ where $a$ is the sound speed $\sqrt{\gamma p / \rho}$, are constant. Thus the states $\rho, u, v, p$ that can be connected to the state $\rho_{L}, u_{L}, v_{L}, p_{L}$ through a 1-rarefaction have to satisfy :

$$
\frac{p}{\rho^{\gamma}}=\frac{p_{L}}{\rho_{L}^{\gamma}} \quad ; \quad u+\frac{2 a}{\gamma-1}=u_{L}+\frac{2 a_{L}}{\gamma-1}
$$

and

$$
v=v_{L}
$$

Combining the two equations (31), we obtain the 1-rarefaction curve that defines in the $(u, p)$ plane the states that can be connected to $q_{L}$ :

$$
u=u_{L}+\frac{2 a_{L}}{\gamma-1}\left(1-\left(\frac{p}{p_{L}}\right)^{\alpha}\right) \quad \text { with } \quad \alpha=(\gamma-1) / 2 \gamma \quad \text { and } \quad p<p_{L}
$$

Similarly, writing that the Riemann invariants are constant through a 3-rarefaction, we obtain the 3-rarefaction curve that defines all the possible states that can be connected to $q_{R}$ through a 3-rarefaction :

$$
u=u_{R}-\frac{2 a_{R}}{\gamma-1}\left(1-\left(\frac{p}{p_{R}}\right)^{\alpha}\right) \quad \text { with } \quad p<p_{R}
$$



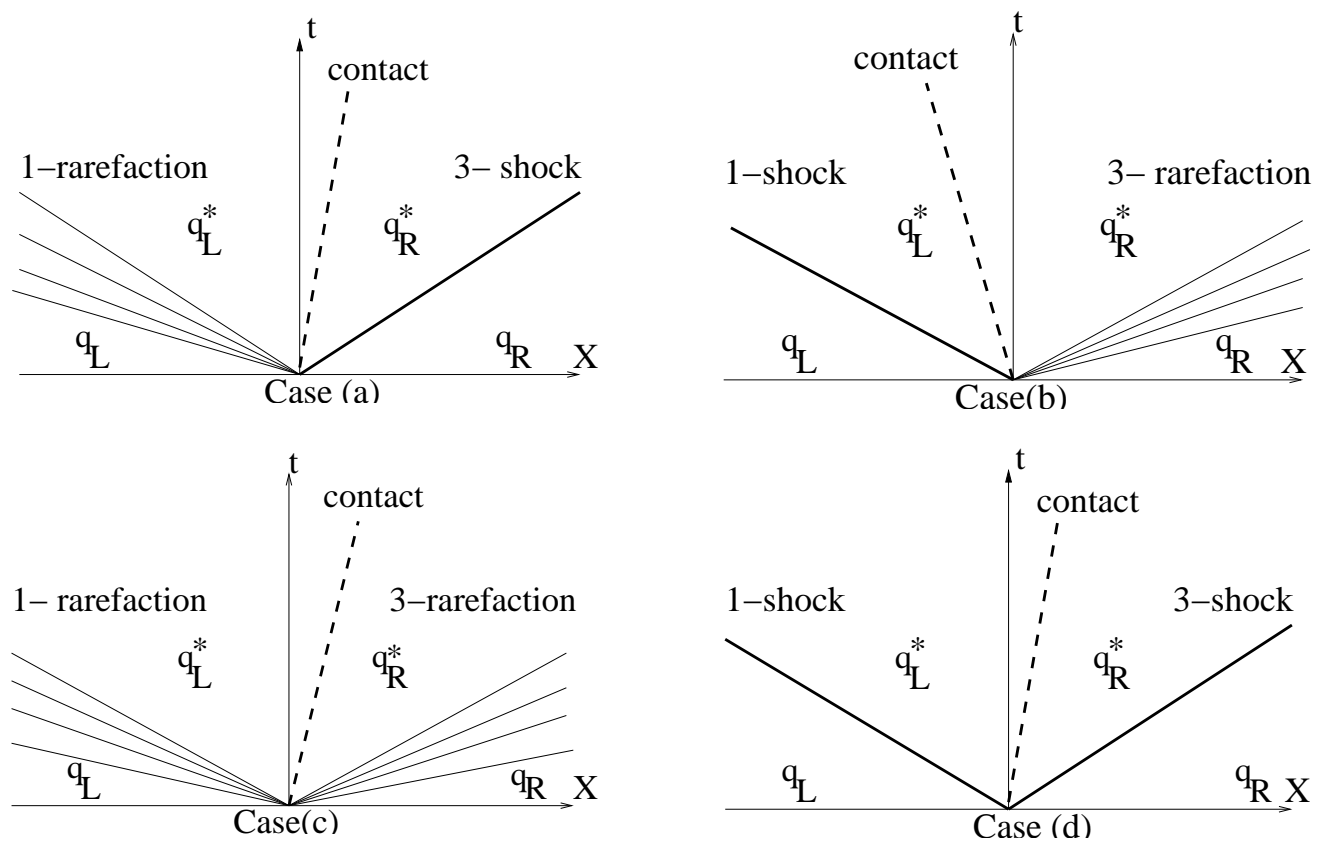

Figure 2: The four possible wave patterns in the solution of the Riemann problem : (a) 1rarefaction, contact, 3-shock, (b) 1-shock, contact, 3-rarefaction, (c) 1-rarefaction, contact, 3rarefaction, (d) 1-shock, contact, 3-shock.

The consideration of the two curves (35)-(37) allows one to compute the solution in case (c) of Fig.2. For the other 3 cases, one has to consider the occurence of shock waves. Writing the Rankine-Hugoniot relations :

$$
\left\{\begin{array}{l}
\Delta \rho u=\sigma \Delta \rho \\
\Delta \rho u^{2}+p=\sigma \Delta \rho u \\
\Delta \rho u v=\sigma \Delta \rho v \\
\Delta(\rho e+p) u=\sigma \Delta \rho e
\end{array}\right.
$$

through the shocks, we obtain the 1-shock and 3-shock curves :

$$
\begin{aligned}
& u=u_{L}-\frac{p-p_{L}}{\sqrt{p+\mu^{2} p_{L}}} \sqrt{\left(1-\mu^{2}\right) / \rho_{L}} \text { with } p>p_{L} \\
& u=u_{R}+\frac{p-p_{R}}{\sqrt{p+\mu^{2} p_{R}}} \sqrt{\left(1-\mu^{2}\right) / \rho_{R}} \text { with } p>p_{R}
\end{aligned}
$$

where $\mu^{2}=(\gamma-1) /(\gamma+1)$. The solution of the Riemann problem is completed by finding the values $\left(u_{*}, p_{*}\right)$ in the states $q_{L *}$ and $q_{R *}$. From an algebraic point of view, this consists to find the intersection of the 1-rarefaction1-shock curve originating from $\left(u_{L}, p_{L}\right)$ with the 3-rarefaction-3-shock curve passing through $\left(u_{R}, p_{R}\right)$. Except in the case of two extremely strong facing rarefactions, these two curves admits a unique intersection point and this completely determines the solution of the Riemann problem.

We are now interested by the solution of this problem in the low Mach number limit. More specifically, we consider initial data $\left(q_{L}, q_{R}\right)$ close to a constant density incompressible flow in the following sense :

$$
\begin{aligned}
& \rho_{L}=\rho_{\text {ref }}\left(\rho_{0}+M_{*}^{2} \rho_{L, 2}+\ldots\right) \\
& u_{L}=a_{r e f}\left(0+M_{*} u_{L, 1}+\ldots\right) \\
& v_{L}=a_{r e f}\left(0+M_{*} v_{L, 1}+\ldots\right) \\
& p_{L}=\rho_{\text {ref }} a_{r e f}^{2}\left(p_{0}+M_{*}^{2} p_{L, 2}+\ldots\right) \\
& \rho_{R}=\rho_{\text {ref }}\left(\rho_{0}+M_{*}^{2} \rho_{R, 2}+\ldots\right) \\
& u_{R}=a_{r e f}\left(0+M_{*} u_{R, 1}+\ldots\right) \\
& v_{R}=a_{r e f}\left(0+M_{*} v_{R, 1}+\ldots\right) \\
& p_{R}=\rho_{r e f} a_{r e f}^{2}\left(p_{0}+M_{*}^{2} p_{R, 2}+\ldots\right)
\end{aligned}
$$


Observe that in accordance with the results that we have recalled in section 2, the initial data are the projection on a piecewise constant field of "well prepared" initial data. In particular, the pressure field does not contain fluctuations of order $M_{*}$. To find the solution of the Riemann problem in the limit $M_{*} \rightarrow 0$, we write the solution in the form :

$$
\begin{aligned}
& \rho=\rho_{\text {ref }}\left(\rho_{0}+M_{*} \rho_{1}+M_{*}^{2} \rho_{2}+\ldots\right) \\
& u=a_{r e f}\left(0+M_{*} u_{1}+\ldots\right) \\
& v=a_{r e f}\left(0+M_{*} v_{1}+\ldots\right) \\
& p=\rho_{r e f} a_{r e f}^{2}\left(p_{0}+M_{*} p_{1}+M_{*}{ }^{2} p_{2}+\ldots\right)
\end{aligned}
$$

Introducing these expressions in the rarefaction and shock curves (35)-(37)-(41)-(43), it is easy to see that in the limit $M_{*} \rightarrow 0$, these curves becomes straigh lines of slopes $\pm 1 / \sqrt{\gamma p_{0} \rho_{0}}$ :

$$
\begin{array}{lll}
\text { 1-Rarefaction } & u_{1}=u_{L, 1}-\frac{1}{\sqrt{\gamma p_{0} \rho_{0}}} p_{1} & \text { for } p_{1}<0 \\
\text { 1-Shock } & u_{1}=u_{L, 1}-\frac{1}{\sqrt{\gamma p_{0} \rho_{0}}} p_{1} & \text { for } p_{1}>0 \\
\text { 3-Rarefaction } & u_{1}=u_{R, 1}+\frac{1}{\sqrt{\gamma p_{0} \rho_{0}}} p_{1} \quad \text { for } p_{1}<0 \\
\text { 3-Shock } & u_{1}=u_{R, 1}+\frac{1}{\sqrt{\gamma p_{0} \rho_{0}}} p_{1} & \text { for } p_{1}>0
\end{array}
$$

As shown in Fig. 3 of the 4 possible wave patterns displayed in Fig. 2, only the 1-rarefaction-3-rarefaction (when $u_{R, 1}-u_{L, 1}>0$ ) and 1-shock-3-shock (when $u_{R, 1}-u_{L, 1}<0$ ) solutions are possible.
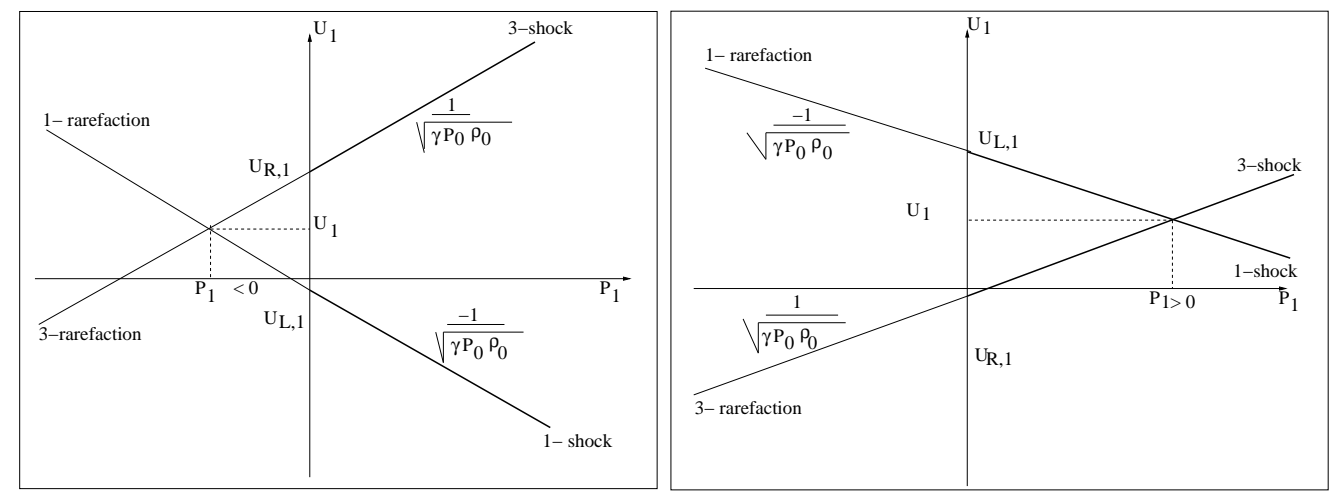

Figure 3: Intersection of rarefaction curves for $u_{R, 1}-u_{L, 1}>0$ (left) and shock curves for $u_{R, 1}-u_{L, 1}<0$ (right).

For these two cases, the computation of the intersection point of the curves (47) gives :

$$
\begin{aligned}
& u_{1}=\left(u_{L, 1}+u_{R, 1}\right) / 2 \\
& p_{1}=-\sqrt{\gamma p_{0} \rho_{0}} \Delta u_{1} / 2
\end{aligned}
$$

with $\Delta u_{1}=\Delta_{R L} u_{1}=u_{R, 1}-u_{L, 1}$. Therefore, it is seen that even if the initial data are of the form (44)-(45) with $p_{L, 1}=p_{R, 1}=0$, the interface pressure contains a fluctuation of order $M_{*}$ :

$$
p=\rho_{\text {ref }} a_{r e f}^{2}\left(p_{0}-\frac{M_{*}}{2} \sqrt{\gamma p_{0} \rho_{0}} \Delta u_{1}+\ldots\right.
$$

Actually, this pressure fluctuation is purely of acoustic origin. The shock curves of (47) are the RankineHugoniot relations for an acoustic system and (48) is in fact the solution of the linear acoustic problem defined by :

$$
\frac{\partial}{\partial t}\left(\begin{array}{c}
u_{1} \\
p_{1}
\end{array}\right)+\left(\begin{array}{cc}
0 & 1 / \rho_{0} \\
\gamma p_{0} & 0
\end{array}\right) \frac{\partial}{\partial x}\left(\begin{array}{c}
u_{1} \\
p_{1}
\end{array}\right)=0
$$


and therefore, the flux computed at the interface instead of being an approximation of the "incompressible flux" is to leading order the flux coming from an acoustic problem generated by the artificial discontinuties at the interfaces between the cells.

To illustrate this analysis, we show in Fig. 4, the solution of the Riemann problem (28) compared to the asymptotic expression (48) for initial data of the form (44)-(45) defined by :

$$
\left\{\begin{array} { l } 
{ \rho _ { L } = 1 . } \\
{ u _ { L } = 1 . } \\
{ v _ { L } = 1 . } \\
{ p _ { L } = 1 0 0 0 1 . }
\end{array} \quad \text { and } \quad \left\{\begin{array}{l}
\rho_{R}=1 . \\
u_{R}=1.75 \text { or } 0.25 \\
v_{R}=1.45 \\
p_{R}=10001.85
\end{array}\right.\right.
$$

The reference Mach number for this case is of order $10^{-2}$. In Fig. 4, the left plots display the case of 2 rarefaction solution (when $u_{R}>u_{L}$ ) while the right plots shows the case of a two shock solution (when $u_{R}<u_{L}$ ). Observe than in the two cases, the pressure jump between the two initial states is extremely small (of the order of $0.01 \%$ ) but the velocity jump creates an extremely large variation of the interface pressure.

From an examination of the Riemann problem in the limit $M_{*} \rightarrow 0$, we can therefore conclude that even if the initial data are close to a constant density incompressible field, the field computed by an upwind solver contains after one time step, acoustic pressure waves of order $M_{*}$ that are much larger than the pressure fluctuations due to the incompressible component. These acoustic components remain present in the following time steps and the equilibrium state of the discrete equations (if it exists) can be described as a balance of acoustic waves instead of representing an approximation of an incompressible field. In section 6 , we present this limit system, the reader will notice the close resemblance of this system with the limit equations obtained from an asymptotic analysis of the Roe scheme that we have presented in [7].
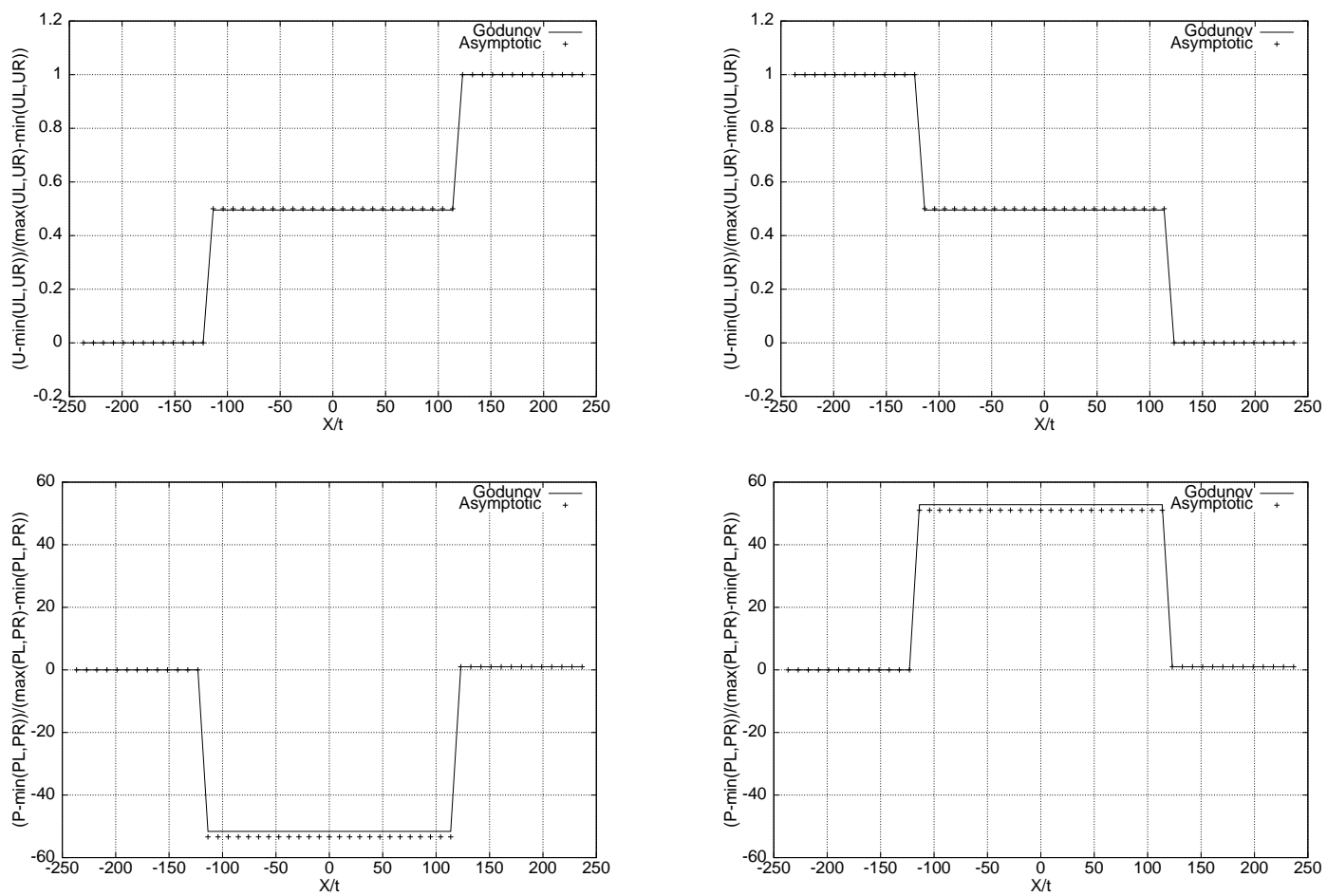

Figure 4: Comparison between Godunov solver and asymptotic analysis for $u_{R}>u_{L}$ (left) and for $u_{R}<u_{L}$ (right). 


\section{The effect of preconditioning}

For Roe type schemes, we have shown in [7] that for very subsonic flows a clear improvement of the accuracy can be obtained by modifying the numerical flux (1) in the following way :

$$
\Phi\left(q_{L}, q_{R}, \mathbf{n}_{L R}\right)=\frac{1}{2}\left(\mathbf{F} \cdot \mathbf{n}_{L R}\left(q_{L}\right)+\mathbf{F} \cdot \mathbf{n}_{L R}\left(q_{R}\right)+P^{-1}\left|P \frac{\partial \mathbf{F} \cdot \mathbf{n}_{L R}}{\partial q}\right| \Delta_{L R} q\right)
$$

where $P$ is a preconditioning matrix. In this section, we propose a similar strategy to overcome the accuracy problem encountered by Godunov type schemes.

More specifically, we will apply preconditioning to the VFRoe class of schemes that we describe now. The VFRoe schemes [10] use a flux of the form :

$$
\Phi\left(q_{L}, q_{R}, \mathbf{n}_{L R}\right)=\mathbf{F} \cdot \mathbf{n}_{L R}\left(q_{L R}\right)
$$

where $q_{L R}$ is a state computed by the solution of a Riemann problem between the two states $q_{L}, q_{R}$. If this Riemann problem is defined by the non linear Euler equations, the scheme defined by (53) is simply Godunov scheme. However, from a computational point of view, it may be interesting to define this Riemann problem by a linearized problem between the two states $\left(q_{L}, q_{R}\right)$ :

$$
\begin{aligned}
& \frac{\partial \tilde{q}}{\partial t}+<A>\frac{\partial \tilde{q}}{\partial x}=0 \\
& \tilde{q}(x, 0)=\left\{\begin{array}{lll}
\tilde{q}=\tilde{q}_{L} & \text { if } & x<0 \\
\tilde{q}=\tilde{q}_{R} & \text { if } & x>0
\end{array}\right.
\end{aligned}
$$

where $\langle A\rangle$ is a constant matrix and $\tilde{q}$ can be the conservative variables $q$ or any other set of independant variables. Here, for instance, we will use the "entropic" variables $\tilde{q}=(p, u, v, s)^{t}$ and define $\langle A\rangle$ by :

$$
<A>=\left[\begin{array}{cccc}
<u\rangle & \gamma\langle p\rangle & 0 & 0 \\
1 /\langle\rho\rangle & \langle u\rangle & 0 & 0 \\
0 & 0 & <u> & 0 \\
0 & 0 & 0 & \langle u\rangle
\end{array}\right]
$$

where $<.>=\left((.)_{L}+(.)_{R}\right) / 2$ denote the arithmetic average between $(\tilde{)})_{L}$ and $(\tilde{.})_{R}$. Thus in $(53), q_{L R}$ is the conservative variables corresponding to $\tilde{q}_{L R}$ solution of the linearized Riemann problem (54). An asymptotic analysis of this upwind scheme similar to the analysis presented in section 3 yields the same conclusions as Godunov scheme. It shows that the interface pressure is given by expression (49) and thus that the pressure field contains fluctuations of order Mach.

In the spirit of the preconditioning of Roe scheme (52), we propose to modify the interface flux $\Phi\left(q_{L}, q_{R}, \mathbf{n}_{L R}\right)=$ F.n $\mathbf{n}_{L R}\left(q_{L R}\right)$ by computing $q_{L R}$ as the solution of a preconditioned Riemann problem. More specifically, in (53) we take $q_{L R}$ as the solution of the preconditioned problem :

$$
\begin{aligned}
& \frac{\partial \tilde{q}}{\partial t}+P<A>\frac{\partial \tilde{q}}{\partial x}=0 \\
& \tilde{q}(x, 0)=\left\{\begin{array}{lll}
\tilde{q}=\tilde{q}_{L} & \text { if } & x<0 \\
\tilde{q}=\tilde{q}_{R} & \text { if } & x>0
\end{array}\right.
\end{aligned}
$$

where $P$ is the preconditioning matrix proposed in [19]

$$
P=\left[\begin{array}{cccc}
\beta^{2} & 0 & 0 & 0 \\
0 & 1 & 0 & 0 \\
0 & 0 & 1 & 0 \\
0 & 0 & 0 & 1
\end{array}\right]
$$

with $\beta$ a parameter of the order of the Mach number : $\beta=M_{*} \beta_{1}$ with $\beta_{1}=\mathcal{O}(1)$. 
We proceed now to show that the interface pressure solution of the preconditioned Riemann problem (56) does not contain fluctuations of order $M_{*}$ if the initial pressure contains only pressure fluctuations of order $M_{*}{ }^{2}$. The left and right states are then defined by :

$$
\begin{aligned}
& \rho_{L}=\rho_{r e f}\left(\rho_{L, 0}+M_{*} \rho_{L, 1}+M_{*}^{2} \rho_{L, 2}+\ldots\right) \\
& u_{L}=a_{r e f}\left(0+M_{*} u_{L, 1}+\ldots\right) \\
& v_{L}=a_{r e f}\left(0+M_{*} v_{L, 1}+\ldots\right) \\
& p_{L}=\rho_{r e f} a_{r e f}^{2}\left(p_{0}+M_{*}^{2} p_{L, 2}+\ldots\right) \\
& \rho_{R}=\rho_{r e f}\left(\rho_{R, 0}+M_{*} \rho_{R, 1}+M_{*}^{2} \rho_{R, 2}+\ldots\right) \\
& u_{R}=a_{r e f}\left(0+M_{*} u_{R, 1}+\ldots\right) \\
& v_{R}=a_{r e f}\left(0+M_{*} v_{R, 1}+\ldots\right) \\
& p_{R}=\rho_{r e f} a_{r e f}^{2}\left(p_{0}+M_{*}^{2} p_{R, 2}+\ldots\right)
\end{aligned}
$$

Note that in (58-59), we allow the presence of density discontinuities of order $\mathcal{O}(1)$ but that the pressure jump between the left and right states is of order $M_{*}^{2}$.

To obtain an explicit expression for the solution of (56), we diagonalize the matrix $\langle A\rangle$. Its eigenvalues are given by :

$$
\begin{aligned}
& \lambda_{1}=\left[\left(1+\beta^{2}\right)<u>-\sqrt{X}\right] / 2 \\
& \lambda_{2}=\lambda_{3}=<u> \\
& \lambda_{4}=\left[\left(1+\beta^{2}\right)<u>+\sqrt{X}\right] / 2
\end{aligned}
$$

with $X=\left[\left(1-\beta^{2}\right)<u>\right]^{2}+4 \beta^{2} \gamma<p>/<\rho>$ and the associated eigenvectors $R_{k}$ are :

$$
R_{1}=\left[\begin{array}{c}
1 \\
\frac{\lambda_{1}-\beta^{2}<u>}{\beta^{2} \gamma<p>} \\
0 \\
0
\end{array}\right], R_{2}=\left[\begin{array}{l}
0 \\
0 \\
0 \\
1
\end{array}\right], R_{3}=\left[\begin{array}{l}
0 \\
0 \\
1 \\
0
\end{array}\right], R_{4}=\left[\begin{array}{c}
1 \\
\frac{\lambda_{4}-\beta^{2}<u>}{\beta^{2} \gamma<p>} \\
0 \\
0
\end{array}\right]
$$

Finally, let us introduce the coordinates $\alpha_{k}\left(\Delta_{R L} \tilde{q}\right)$ of the jump $\Delta_{R L} \tilde{q}=\tilde{q}_{R}-\tilde{q}_{L}$ in the basis of the eigenvectors $R_{k}$ :

$$
\begin{aligned}
& \alpha_{1}=-\frac{\left(\lambda_{1}-<u>\right) \Delta p+\beta^{2} \gamma<p>\Delta u}{\sqrt{X}} \\
& \alpha_{2}=\Delta S \\
& \alpha_{3}=\Delta v \\
& \alpha_{4}=\frac{\left(\lambda_{4}-<u>\right) \Delta p+\beta^{2} \gamma<p>\Delta u}{\sqrt{X}}
\end{aligned}
$$

then, the solution of (56) is given by 4 constant states $\tilde{q}_{L}, \tilde{q}_{L}^{*}, \tilde{q}_{R}^{*}, \tilde{q}_{R}$ defined by :

$$
\tilde{q}(x, t)= \begin{cases}\tilde{q}_{L} & \text { for } \quad x / t<\lambda_{1} \\ \tilde{q}_{L}^{*}=\tilde{q}_{L}+\alpha_{1} R_{1} & \text { for } \quad \lambda_{1}<x / t<\lambda_{2} \\ \tilde{q}_{R}^{*}=\tilde{q}_{R}-\alpha_{4} R_{4} & \text { for } \quad \lambda_{2}<x / t<\lambda_{4} \\ \tilde{q}_{R} & \text { for } \quad \lambda_{4}>x / t\end{cases}
$$

Using the expressions of the left and right eigenvectors (62)-(61), together with the asymptotic expansions (58)-(59), we obtain the following asymptotic expressions for the two states $\tilde{q}_{L}^{*}, \tilde{q}_{R}^{*}$ : 
(Note that the value of $u$ and $p$ are equal in the two states $\tilde{q}_{L}^{*}, \tilde{q}_{R}^{*}$ )

$$
\begin{aligned}
u_{*}=a_{r e f}\left(0+M_{*}\left(<u_{1}>-\frac{<u_{1}>}{2 \sqrt{X_{0}}} \Delta u_{1}-\frac{\Delta p_{2}}{<\rho_{0}>\sqrt{X_{0}}}\right)+\right. \\
\left.M_{*}^{2}\left(<u_{2}>-\frac{<u_{1}>}{2 \sqrt{X_{0}}} \Delta u_{2}+(\ldots) \Delta u_{1}+\frac{<\rho_{1}>\Delta p_{2}}{<\rho_{0}>\sqrt{X}_{0}}+(\ldots) \Delta p_{2}\right)+\ldots\right) \\
p^{*}=\rho_{r e f} a_{r e f}^{2}\left(p_{0}+M_{*}^{2}\left(<p_{2}>-\frac{\beta_{1}^{2} \gamma p_{0}}{\sqrt{X_{0}}} \Delta u_{1}+\frac{<u_{1}>}{2 \sqrt{X_{0}}} \Delta p_{2}\right)+\ldots\right)
\end{aligned}
$$

where $X_{0}=\left(<u_{1}>\right)^{2}+4 \beta_{1}{ }^{2} \gamma p_{0} /<\rho_{0}>,<.>=\left((.)_{L}+(.)_{R}\right) / 2$ denotes the arithmetic average and (..) stands for some complex terms that are not explicited here.

The values of the densities and tangential velocities are given by :

$$
\begin{aligned}
\rho_{L}^{*} & =\rho_{\text {ref }}\left(\rho_{L, 0}+M_{*} \rho_{L, 1}+\right. \\
& \left.M_{*}{ }^{2}\left(\rho_{L, 2}-\frac{\beta_{1}{ }^{2} \rho_{L, 0}}{\sqrt{X_{0}}} \Delta u_{1}+\frac{\rho_{L, 0}<u_{1}>}{2 \gamma p_{0} \sqrt{X_{0}}} \Delta p_{2}+\frac{\rho_{L, 0}}{2 \gamma p_{0}} \Delta p_{2}\right)+\ldots\right) \\
v_{L}^{*} & =v_{L} \\
\rho_{R}^{*} & =\rho_{r e f}\left(\rho_{R, 0}+M_{*} \rho_{R, 1}+\right. \\
& \left.M_{*}{ }^{2}\left(\rho_{R, 2}-\frac{\beta_{1}{ }^{2} \rho_{R, 0}}{\sqrt{X_{0}}} \Delta u_{1}+\frac{\rho_{R, 0}<u_{1}>}{2 \gamma p_{0} \sqrt{X_{0}}} \Delta p_{2}-\frac{\rho_{R, 0}}{2 \gamma p_{0}} \Delta p_{2}\right)+\ldots\right) \\
v_{R}^{*} & =v_{R}
\end{aligned}
$$

From expression (64), it is seen that the interface pressure is free of order Mach fluctuations.

Now, let us return to the numerical experiments that we have presented in the introduction. Fig. 6 presents the pressure fields for the same three decrasing Mach number than in Fig. 1. We see, that in contrast to the results obtained with the original fluxes, the solution converge to an unique solution.

Fig. 5 presents the pressure fluctuations with respect to the Mach number. As implied by expression (64), the pressure fluctuations scale exactely with the square of the Mach number in agreement with the behavior of the continuous equations in the case of well-prepared initial data.

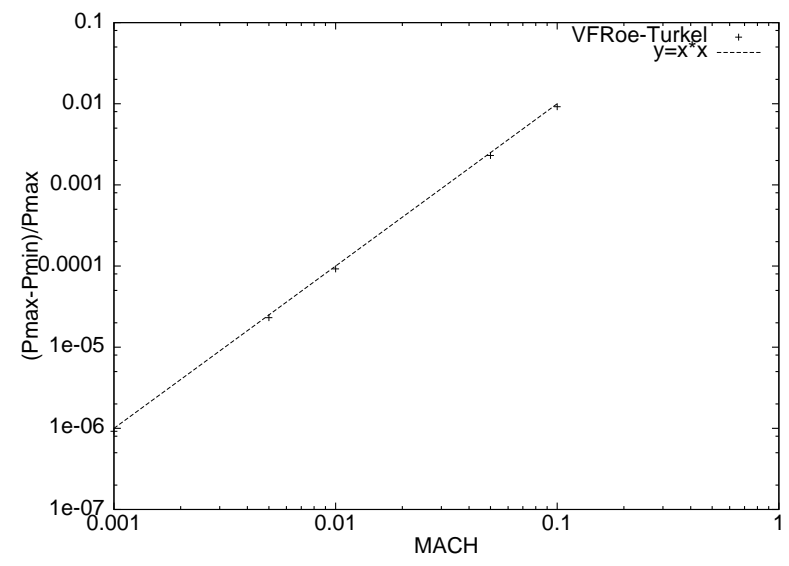

Figure 5: Pressure fluctuations vs inflow Mach number ; for comparison the curve $\mathrm{y}=\mathrm{x}^{*} \mathrm{x}$ is displayed. 

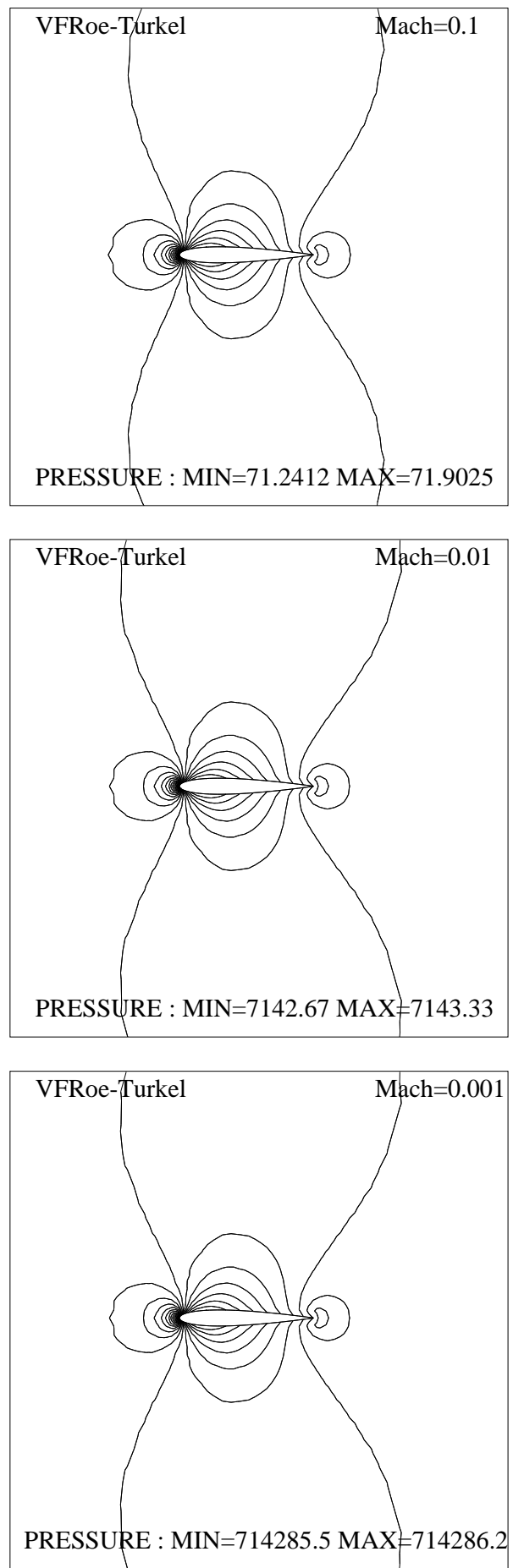

Figure 6: Isovalues of the pressure, on a 3114 node mesh for $M_{\infty}=0.1$ (top), $M_{\infty}=0.01$ (middle), $M_{\infty}=0.001$ (bottom). 


\section{Conclusion}

Using an asymptotic analysis of Godunov scheme, we have exhibited the mechanism that prevents this type of approximation to compute near incompressible flows. The trouble comes from the fact that the interface pressure computed by the Riemann solver contains pressure fluctuations of order Mach even if the initial data that define the Riemann problem contain fluctuations that scale with the square of the Mach number. This fact is deeply linked to the behavior of the solutions of the Euler equations at low Mach number : if the initial data are not well prepared, then in addition to the incompressible component, the solutions of the Euler equations contain also an extremely fast acoustic part. This acoustic part is computed by the discrete scheme that reacts to the artificial discontinuities due to the projection of the initial data on piecewise constants by creating acoustic waves at the interface between the cells.

We also show that as in the case of Roe scheme [7], this situation can be controlled by modifying the numerical fluxes with the help of preconditioning techniques. More specifically, we propose to change the original Riemann problem by a preconditioned Riemann problem. We apply this strategy to a VFRoe scheme. In this latter case, we show both theoretically and numerically that the solution of the preconditioned Riemann problem possesses pressure fluctuations of the correct magnitude.

Preconditioned compressible solvers [7], [2], [3] are able to deal with the singularity of the Euler equations in the low Mach number regime. Comparisons with incompressible and low Mach number solvers show the excellent behavior of these methods (see e.g. [24], [13], [14], [15]). However, in our opinion, the reason of this good behavior remains unclear. As mentioned by B. van Leer and D-L. Darmofal in [23] the beneficial influence of preconditioning on the accuracy of compressible solvers in the low Mach number regime was an unexpected side effect. In this paper, as a first step in the analysis of these methods, we have shown that the most obvious result of preconditioning consists of removing the fluctuations of order Mach in the interface pressures. Thus, in some sense, the preconditioned solvers suppress the unwanted acoustic waves generated by the interface discontinuities. It will remain to prove that the resulting fluxes provides a good approximation of the incompressible system. While there are experimental evidences showing that this is indeed the case, the theoretical proof of this fact remains to be found. 


\section{Annex I}

Here as in section 3, we consider the limit solution of the Riemann problem (28) in order to identify the limit system that the discrete unknowns satisfy. In section 3, we have shown that even if the initial data are "well prepared" and of the form (44-45), the solution after one time step contains pressure waves of order $M_{*}$. Therefore, we have to consider more general initial data than (44-45), i.e we have to consider the case where the initial data although still characterized by a low Mach number are not well prepared. In addition, since the incompressible Euler model supports density discontinuities, we also allow in the initial data, the possibility to have $\mathcal{O}(1)$ density discontinuities. Thus the two states $q_{L}$ and $q_{R}$ are assumed to have the following form :

$$
\begin{aligned}
& \rho_{L}=\rho_{\text {ref }}\left(\rho_{L, 0}+M_{*} \rho_{L, 1}+M_{*}^{2} \rho_{L, 2}+\ldots\right) \\
& u_{L}=a_{\text {ref }}\left(0+M_{*} u_{L, 1}+M_{*}^{2} u_{L, 2}+\ldots\right) \\
& v_{L}=a_{\text {ref }}\left(0+M_{*} v_{L, 1}+M_{*}{ }^{2} v_{L, 2}+\ldots\right) \\
& p_{L}=\rho_{\text {ref }} a_{r e f}^{2}\left(p_{0}+M_{*} p_{L, 1}+M_{*}^{2} p_{L, 2}+\ldots\right)
\end{aligned}
$$

and

$$
\begin{aligned}
& \rho_{R}=\rho_{\text {ref }}\left(\rho_{R, 0}+M_{*} \rho_{R, 1}+M_{*}^{2} \rho_{R, 2}+\ldots\right) \\
& u_{R}=a_{r e f}\left(0+M_{*} u_{R, 1}+M_{*}^{2} u_{R, 2}+\ldots\right) \\
& v_{R}=a_{r e f}\left(0+M_{*} v_{R, 1}+M_{*}^{2} v_{R, 2}+\ldots\right) \\
& p_{R}=\rho_{r e f} a_{r e f}^{2}\left(p_{0}+M_{*} p_{R, 1}+M_{*}^{2} p_{R, 2}+\ldots\right)
\end{aligned}
$$

As in section 3, we introduce these asymptotic expansion in the shock and rarefaction curves. It is easily seen that again these curves degenerate into straight lines of slopes $-1 / \sqrt{\gamma p_{0} \rho_{L, 0}}$ and $+1 / \sqrt{\gamma p_{0} \rho_{R, 0}}$. However, because $p_{L, 1}$ and $p_{R, 1}$ are not zero, the four possible waves pattern of Fig. 2 can occur. Moreover, due to the $\mathcal{O}(1)$ density discontinuities, the speeds of sound are not equal on the two sides of the contact discontinuity. However, in the four possible cases, the intersection of the shock and rarefaction curves gives :

$$
\begin{aligned}
u^{*} & =a_{r e f}\left(0+M_{*}\left(\frac{\sqrt{\rho_{L, 0}} u_{L, 1}+\sqrt{\rho_{R, 0}} u_{R, 1}}{\sqrt{\rho_{L, 0}}+\sqrt{\rho_{R, 0}}}-\frac{1}{\sqrt{\gamma p_{0}}} \frac{\Delta p_{1}}{\sqrt{\rho_{L, 0}}+\sqrt{\rho_{R, 0}}}\right)\right. \\
& \left.+M_{*}^{2} \ldots\right) \\
p^{*} & =\rho_{r e f} a_{r e f}^{2}\left(p_{0}+M_{*}\left(\frac{\sqrt{\rho_{R, 0}} p_{L, 1}+\sqrt{\rho_{L, 0}} p_{R, 1}}{\sqrt{\rho_{L, 0}}+\sqrt{\rho_{R, 0}}}-\sqrt{\gamma p_{0}} \frac{\sqrt{\rho_{L, 0} \rho_{R, 0}}}{\sqrt{\rho_{L, 0}}+\sqrt{\rho_{R, 0}}} \Delta u_{1}\right)\right. \\
& \left.+M_{*}{ }^{2} \ldots\right)
\end{aligned}
$$

where here $\Delta()=.\Delta_{R L}()=.(.)_{R}-(.)_{L}$. The values of the density and tangential velocities on the two sides of the contact discontinuity are given by :

$$
\begin{aligned}
\rho_{L}^{*} & =\rho_{r e f}\left(\rho_{L, 0}+M_{*}\left(\rho_{L, 1}-\rho_{L, 0} \frac{\sqrt{\rho_{L, 0} \rho_{R, 0}}}{\sqrt{\rho_{L, 0}}+\sqrt{\rho_{R, 0}}} \frac{\Delta u_{1}}{\sqrt{\gamma p_{0}}}+\frac{\rho_{L, 0} \sqrt{\rho_{L, 0}}}{\sqrt{\rho_{L, 0}}+\sqrt{\rho_{R, 0}}} \frac{\Delta p_{1}}{\gamma p_{0}}\right)\right. \\
& \left.+M_{*}{ }^{2} \cdots\right) \\
v_{L}^{*} & =v_{L} \\
\rho_{R}^{*} & =\rho_{r e f}\left(\rho_{R, 0}+M_{*}\left(\rho_{R, 1}-\rho_{R, 0} \frac{\sqrt{\rho_{L, 0} \rho_{R, 0}}}{\sqrt{\rho_{L, 0}}+\sqrt{\rho_{R, 0}}} \frac{\Delta u_{1}}{\sqrt{\gamma p_{0}}}+\frac{\rho_{R, 0} \sqrt{\rho_{R, 0}}}{\sqrt{\rho_{L, 0}}+\sqrt{\rho_{R, 0}}} \frac{\Delta p_{1}}{\gamma p_{0}}\right)\right. \\
& \left.+M_{*}{ }^{2} \ldots\right) \\
v_{R}^{*} & =v_{R}
\end{aligned}
$$

Consider now the application of Godunov scheme in a Finite Volume framework. For simplicity, we consider that we use a regular cartesian grid of uniform mesh size $\delta$ in two dimensions. $i=(i, j)$ is the index of the node whose coordinates are $(i \delta, j \delta)$ and we use the notation $\mathcal{V}(i)=\{(i-1, j),(i+1, j),(i, j-1),(i, j+1)\}$ or $\mathcal{V}(\boldsymbol{i})=\{N, S, E, W\}$ for labelling the neighbors of the grid node $\boldsymbol{i}$. The cell associated with node $\boldsymbol{i}$ is $C_{\boldsymbol{i}}=[(i-1 / 2) \delta,(i+1 / 2) \delta] \times[(j-1 / 2) \delta,(j+1 / 2) \delta], \vec{n}$ is the outward unit normal vector on $\partial C_{\boldsymbol{i}}$ and we note $\vec{n}_{i l}=\frac{\int_{C_{i} \cap C_{l}} \vec{n}}{\delta}$. 
When a steady state is reached, the application of a first-order finite volume scheme yields the following semi-discrete equation :

$$
\sum_{\boldsymbol{l} \in \mathcal{V}(\boldsymbol{i})} \vec{F}\left(q_{i l}\right) \cdot \vec{n}_{i l}=0
$$

where $q_{i l}$ denote the value of the state on the interfaces between the cells $i$ and $\boldsymbol{l}$. Following the analysis that we have performed on the asymptotic solution of the Riemann problem, we write these interface values in the following form:

$$
\begin{aligned}
& \rho_{\boldsymbol{i l}}=\rho_{\text {ref }} \quad\left(\rho_{\boldsymbol{i l}}^{0}+M_{*} \rho_{\boldsymbol{i l}}^{1}+M_{*}^{2} \rho_{\boldsymbol{i l}}^{2}+\ldots\right) \\
& u_{i l}=a_{r e f} \quad\left(0+\quad M_{*} u_{i l}^{1}+M_{*}^{2} u_{i l}^{2}+\ldots\right) \\
& v_{\boldsymbol{i l}}=a_{r e f} \quad\left(0+\quad M_{*} v_{i l}^{1}+\quad M_{*}^{2} v_{i l}^{2}+\ldots\right) \\
& p_{i l}=\rho_{\text {ref }} a_{\text {ref }}^{2}\left(p_{i l}^{0}+M_{*} p_{i l}^{1}+\quad M_{*}^{2} p_{i l}^{2}+\ldots\right)
\end{aligned}
$$

then the flux function evaluated on this state has the following expansion (here $u_{\boldsymbol{i l}}, v_{\boldsymbol{i l}}$ denote respectively the normal and tangential velocities on the interface):

$$
\begin{aligned}
& \left(\vec{F}\left(q_{i l}\right) \cdot \vec{n}_{\boldsymbol{i l}}\right)_{1}=\rho_{\text {ref }} a_{r e f}\left(M_{*} \rho_{\boldsymbol{i l}}^{0} u_{\boldsymbol{i l}}^{1}+\mathcal{O}\left(M_{*}^{2}\right)\right) \\
& \left(\vec{F}\left(q_{\boldsymbol{i l}}\right) \cdot \vec{n}_{\boldsymbol{i l}}\right)_{2}=\rho_{\text {ref }} a_{r e f}^{2}\left(p_{\boldsymbol{i l}}^{0}+M_{*} p_{\boldsymbol{i l}}^{1}+M_{*}^{2}\left(\rho_{\boldsymbol{i l}}^{0}\left(u_{\boldsymbol{i l}}^{1}\right)^{2}+p_{\boldsymbol{i l}}^{2}\right)+\mathcal{O}\left(M_{*}^{3}\right)\right) \\
& \left(\vec{F}\left(q_{\boldsymbol{i l}}\right) \cdot \vec{n}_{\boldsymbol{i l}}\right)_{3}=\rho_{r e f} a_{r e f}^{2}\left(M_{*}^{2} \rho_{\boldsymbol{i l}}^{0} u_{\boldsymbol{i l}}^{1} v_{\boldsymbol{i} \boldsymbol{l}}^{1}+\mathcal{O}\left(M_{*}^{3}\right)\right) \\
& \left(\vec{F}\left(q_{\boldsymbol{i l}}\right) \cdot \vec{n}_{\boldsymbol{i l}}\right)_{4}=\rho_{r e f} a_{r e f}^{3}\left(M_{*} \frac{\gamma}{\gamma-1} p_{\boldsymbol{i l}}^{0} u_{\boldsymbol{i l}}^{1}+\mathcal{O}\left(M_{*}^{2}\right)\right)
\end{aligned}
$$

From the expression (68) of the interface pressure, it is seen that $p_{\boldsymbol{i l}}^{0}=p^{0} \forall \boldsymbol{i l}$ and then the order 0 momentum equations degenerates into :

$$
p^{0} \sum_{\boldsymbol{l} \in \mathcal{V}(\boldsymbol{i})} \vec{n}_{\boldsymbol{i l}}=0
$$

but the order $M_{*}$ momentum equations are:

$$
\sum_{\boldsymbol{l} \in \mathcal{V}(\boldsymbol{i})} p_{i l}^{1} \vec{n}_{i l}=0
$$

where the value of $p_{\boldsymbol{i l}}^{1}$ is given in (68). This gives for the horizontal momentum equation :

$$
\begin{aligned}
& -\frac{\sqrt{\rho_{\boldsymbol{i}}^{0}} p_{W}^{1}+\sqrt{\rho_{W}^{0}} p_{i}^{1}}{\sqrt{\rho_{\boldsymbol{i}}^{0}}+\sqrt{\rho_{W}^{0}}}+\frac{\sqrt{\rho_{E}^{0}} p_{\boldsymbol{i}}^{1}+\sqrt{\rho_{\boldsymbol{i}}^{0}} p_{E}^{1}}{\sqrt{\rho_{\boldsymbol{i}}^{0}}+\sqrt{\rho_{E}^{0}}} \\
& =\sqrt{\gamma p^{0}}\left(\frac{\sqrt{\rho_{\boldsymbol{i}}^{0} \rho_{E}^{0}}}{\sqrt{\rho_{\boldsymbol{i}}^{0}}+\sqrt{\rho_{E}^{0}}} \Delta_{E} \boldsymbol{i}^{1}-\frac{\sqrt{\rho_{\boldsymbol{i}}^{0} \rho_{W}^{0}}}{\sqrt{\rho_{\boldsymbol{i}}^{0}}+\sqrt{\rho_{W}^{0}}} \Delta_{\boldsymbol{i}} u^{1}\right)
\end{aligned}
$$

and a similar expression for the vertical momentum equation. From (73), it is clear that $p^{1}$ is not 0 as soon as the velocity field is not uniform. Note also that equation (73) is almost identical to the equation obtained for Roe discretisation in [7]. 


\section{References}

[1] H. Bijl and P Wesseling. A unified method for computing incompressible and compressible flows in boundary fitted coordinate. J. Comput. Phys, 141:153-173, 1998.

[2] S. Clerc. Numerical simulation of the homogeneous equilibrium for two-phase flows. Journal of Computational Physics, 161:354-375, 2000.

[3] A. Dervieux, D. Guezengar, Hervé Guillard, and C. Viozat. Analysis of low Mach simulations with compressible upwind codes. In ECCOMAS 98. John Wiley and Sons, 1998.

[4] R. Radespiel E. Turkel and N. Kroll. Assessment of preconditioning methods for multidimensional aerodynamics. Computers and Fluids, 26:613-634, 1997.

[5] E. Grenier. Oscillatory perturbations of the Navier-Stokes equations. J. Math. Pures. Appl., 76:477-498, 1997.

[6] H. Guillard and R. Abgrall. Modélisation des fluides numériques compressibles, volume 5 of SERIES IN APPLIED MATHEMATIC. Gauthier-Villars-North Holland, Paris, 2001.

[7] H. Guillard and C. Viozat. On the behavior of upwind schemes in the low Mach number limit. Computers and fluids, 28:63-96, 1999.

[8] S. Klainerman and A. Majda. Compressible and incompressible fluids. Communications on Pure and Applied Mathematics, 35:629-653, 1982.

[9] H.-O. Kreiss. Problems with different time scales for partial differential equations. Communications on Pure and Applied Mathematics, 33:399-440, 1980.

[10] J.M. Masella, I. Faille, and T. Galouët. On an approximate Godunov scheme. Int. J. of Comp. Fluid Dyn., 12:133-149, 1999.

[11] G. Metivier and S. Schochet. The incompressible limit of the nonisentropic Euler equations. Arch. Rat. Mech. Anal., 2000. to be published.

[12] G. Metivier and S. Schochet. Limite incompressible des équations d'Euler non-isentropiques. Séminaire équations aux dérivées partielles de l'école Polytechnique, to be found at : http://www.maths.univrennes1.fr/ metivier/preprints.html, 2001.

[13] H. Paillere, S. Clerc, C. Viozat, I. Toumi, and J-P. Magnaud. Numerical methods for low Mach number thermal-hydraulic flows. In ECCOMAS 98. John Wiley and Sons, 1998.

[14] H. Paillère, C. Viozat, A. Kumbaro, and I. Toumi. Comparison of low Mach number models for natural convection problems. In Eurotherm Seminar No 63. Genoa, Italy, 6-8 September 1999.

[15] E. Schall, C. Viozat, B. Koobus, and A. Dervieux. On the computation of unsteady ans steady low Mach flows with implicit upwind methods. In ECCOMAS 2001, 2001.

[16] S. Schochet. Asymptotics for symmetric hyperbolic systems with a large parameter. Journal of differential equations, 75:1-27, 1988.

[17] S. Schochet. Fast singular limits of hyperbolic pdes. Journal of differential equations, 114:476-512, 1994.

[18] E.F. Toro. Riemann Solvers and numerical methods for fluid dynamics. Springer Verlag, Berlin, 1997.

[19] E. Turkel. Preconditioned methods for solving the incompressible and low speed compressible equations. J. Comput. Phys, 72:277-298, 1987.

[20] E. Turkel. Review of preconditioning methods for fluid dynamics. Applied Numerical Mathematics, 12:257$284,1993$.

[21] E. Turkel, A. Fiterman, and B. van Leer. Preconditioning and the limit of the compressible to the incompressible flow equations for finite difference schemes, pages 215-234. John Wiley and Sons, Chichester, 1994. 
[22] S. Ukai. The incompressible limit and the initial layer of the compressible Euler equations. J. Math. Kyoto Univ., 26:323-331, 1986.

[23] B. van Leer and D-L. Darmofal. Steady Euler Solutions in 0(n) Operations. In J. Vierendeels E. Dick, K. Riemslagh, editor, Multigrid methods VI : proceedings of the Sixth European multigrid conference, held in Gent, Belgium, September 27-30, 1999, pages 24-33. Springer, 2000. Lecture notes in computational science and engineering; 14.

[24] C. Viozat. Calcul d'écoulements stationnaires et instationnaires à petit nombre de Mach et en maillages étirés. PhD thesis, University of Nice-Sophia-Antipolis, 1998.

[25] C. Viozat. Calcul d'écoulements diphasiques dans une tuyère : Influence de la renormalisation du schéma de flux. Technical Report SYSCO/LGLS/RT/00-014, CEA, 2000.

[26] G. Volpe. Performance of compressible flow codes at low Mach number. AIAA Journal, 31:49-56, 1993.

[27] O.C. Zienkiewicz, J. Szmelter, and J. Perraire. Compressible and incompressible flows : An algorithm for all seasons. Comput. Methods Appl. Mech. Eng., 78(1):105-121, 1990. 


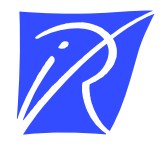

Unité de recherche INRIA Sophia Antipolis 2004, route des Lucioles - B.P. 93 - 06902 Sophia Antipolis Cedex (France)

Unité de recherche INRIA Lorraine : Technopôle de Nancy-Brabois - Campus scientifique 615, rue du Jardin Botanique - B.P. 101 - 54602 Villers lès Nancy Cedex (France)

Unité de recherche INRIA Rennes : IRISA, Campus universitaire de Beaulieu - 35042 Rennes Cedex (France)

Unité de recherche INRIA Rhône-Alpes : 655, avenue de l'Europe - 38330 Montbonnot St Martin (France)

Unité de recherche INRIA Rocquencourt : Domaine de Voluceau - Rocquencourt - B.P. 105 - 78153 Le Chesnay Cedex (France)

Éditeur

INRIA - Domaine de Voluceau - Rocquencourt, B.P. 105 - 78153 Le Chesnay Cedex (France)

http://www.inria.fr

ISSN 0249-6399 